\title{
Gravitational and Experimental Electromagnetic Contributions to Cerebral Effects Upon Deviations from Random Number Variations Generated by Electron Tunneling
}

\author{
Joey M. Caswell ${ }^{\star}$, Mark W. G. Collins ${ }^{\star *}$, David A. E. Vares ${ }^{\star \star *}$, \\ Lyndon M. Juden-Kelly****, Michael A. Persinger***** \\ Laurentian University, 935 Ramsey Lake Road, Sudbury P3E 2C6, Ontario, Canada \\ *-*****E-mails address: jx_caswell@laurentian.ca , mw_collins@laurentian.ca , \\ dx_vares@laurentian.ca,Ix_judenkelly@laurentian.ca , \\ mpersinger@laurentian.ca
}

\begin{abstract}
New theoretical and traditional quantitative solutions involving a pervasive unit quantum of $\sim 10^{-20} \mathrm{~J}$ within biological and large-scale physical systems predicted that the mass of the human subject, subtle changes in gravitational phenomena, and the energy available within the cerebral volume should affect proximal random number variations produced by electron tunneling. In a series of experiments application of a specific, physiologically-patterned weak magnetic field over the right temporal lobe significantly enhanced the effects of intention upon deviations from random variations created by electron tunneling devices at a distance of $1 \mathrm{~m}$. These variations were strongly $(\mathrm{r} \sim 0.80)$ correlated with the coupling between the forces from the background free oscillations of the earth and the energy differences across the cell width between lunar perigee and apogee. The results support the approach that complex cognitive processes including "intention" can be described by physicochemical parameters and their magnitude of energies are within the range by which interactions or modulations from subtle gravitational forces applied across the cellular membrane and width might occur.
\end{abstract}

Keywords: gravitational energy; random numbers; electron tunneling; applied weak magnetic fields; cerebral function; perigee-apogee effects

\section{INTRODUCTION}

Experimental demonstrations of the quantitative interaction between living masses, gravitational phenomena and electromagnetic variations have the capacity to reveal the intricate relationships between all three of these classes of phenomena within a universal context [1]. A fundamental quantum of energy associated with living systems, as manifested by the plasma membrane, is $\sim 10^{-20} \mathrm{~J}[2]$, which is the increment energy associated with the separation of $\mathrm{K}^{+}$ions correlated with the resting membrane potential, the action potential $(\Delta \mathrm{v}$ $\left.=1.4 \cdot 10^{-1} \mathrm{~V} \cdot 1.6 \cdot 10^{-19} \mathrm{~A} \cdot \mathrm{s}=2.2 \cdot 10^{-20} \mathrm{~J}\right)$, and the value associated with hinge motions 
subsequent to the sequestering of the agonist to the receptor. It is the same effective value for second shell hydrogen bonds that are implicitly involved with proton mobility in water [3]. Approximately $10^{-20} \mathrm{~J}$, when solved for its frequency (by dividing Planck's constant), results in a wavelength that is the average width of a cell $(\sim 10 \mu \mathrm{m})$ which is remarkably congruent with the expected peak wavelength of a $37^{\circ} \mathrm{C}(310 \mathrm{~K})$ black body according to Wien's law.

What is less known is that the gravitational energy from the earth across the membrane of a typical cell $(10 \mu \mathrm{m})$ is also $\sim 10^{-20} \mathrm{~J}$. Assuming an average mass of a cell is $5 \cdot 10^{-13} \mathrm{~kg}$ and the earth's mass is $5.976 \cdot 10^{24} \mathrm{~kg}$, the force at the inverse square of the distance from the earth's center (radius, $6.378 \cdot 10^{6} \mathrm{~m}$ ) multiplied by $\mathrm{G}\left(6.672 \cdot 10^{-11} \mathrm{~m}^{3} \mathrm{~kg}^{-1} \mathrm{~s}^{-2}\right)$ is about $10^{-12} \mathrm{~N}$. When applied across the width of a plasma membrane $\left(10^{-8} \mathrm{~m}\right)$ that separates the ions contributing to the voltage of the resting membrane potential, the energy is $10^{-20} \mathrm{~J}$. Persinger et al [4] calculated that the average force available within the known universe per Planck's voxel (the cube of Planck's length) when spread across the neutral hydrogen line results in a value of $\sim 10^{-20} \mathrm{~J}$.

That changes in geomagnetic activity, as one source of electromagnetic perturbations, could interact with gravitational process has been shown theoretically by Minakov et al [5]. Their equations indicated that the conversion of plane gravity waves into electromagnetic radiation would occur optimally with the primary harmonic of the Schumann resonance which is generated within the earth-ionospheric cavity. Vladmirski [6] measured discrete enhancements of about $10^{-3}$ of the value of $\mathrm{G}$, the gravitational constant, during lower geomagnetic activity. Indicators of equivalence between gravitational and electromagnetic properties appear to be more related to discrete quantitative solutions than to a singular equation [7].

That human masses could interact with fluctuations in gravitational forces through resonance processes and be coupled with adjacent physical chemical reactions has potential. Fundamental spheroidal modes or background free oscillations within the earth occupy a relatively wide band between $2 \mathrm{mHz}$ and $7 \mathrm{mHz}$. Their peak-to-peak amplitudes are in the order of $0.5 \cdot 10^{-11} \mathrm{~m} \cdot \mathrm{s}^{-2}$. According to Nishida et al [8] the excitation source emerges at the boundary between the earth's surface and atmosphere. For a $70 \mathrm{~kg}$ person or the $1.5 \mathrm{~kg}$ human cerebrum this would result in forces of $3.5 \cdot 10^{-10} \mathrm{~N}$ and $7 \cdot 10^{-12} \mathrm{~N}$, respectively. For the latter, if applied across the width of neuronal membrane $\left(10^{-8} \mathrm{~m}\right)$ the energy would be within the range of $10^{-20} \mathrm{~J}$.

However, assuming a cross sectional length of $2.2 \cdot 10^{-1} \mathrm{~m}$ for the whole body and $1.1 \cdot 10^{-}$ ${ }^{1} \mathrm{~m}(11 \mathrm{~cm})$ for the cerebrum the equivalent energies for the $10^{-10}$ and $7 \cdot 10^{-12} \mathrm{~N}$ forces are $7.7 \cdot 10^{-11} \mathrm{~J}$ and $10^{-13} \mathrm{~J}$, respectively. For comparison the magnetic energy within the cerebral volume associated with the physiologically-patterned magnetic fields we have found to be most effective for altering subjective states with correlative measurable changes within the cerebral volume [9] involve a range between $\sim 40 \mathrm{nT}$ and $400 \mathrm{nT}$ amplitudes. From the formula:

$$
\mathrm{J}=\left[\mathrm{B}^{2}(2 \cdot 4 \pi \mu)^{-1}\right] \cdot \mathrm{V}
$$

where $\mathrm{B}$ is the strength of the field, $\mu$ is magnetic permeability and $\mathrm{v}$ is volume $\left(\sim 1.3 \cdot 10^{-3} \mathrm{~m}^{3}\right)$ of the cerebrum, the resultant magnetic energy is within the range of $\sim 10^{-13}$ to $10^{-12} \mathrm{~J}$. In other words the magnetic energies from the experimentally applied fields have the capacity to enhance the processes that reveal the coupling between cerebral activity and subtle gravitational-like energies from the earth. 
These calculated values $\left(\sim 10^{-13} \mathrm{~J}\right)$ are relevant because the energies associated with the activity of only $10^{6}$ neurons within the cerebral cortices each discharging around 7 to $8 \mathrm{~Hz}$ (the intrinsic resonance of the cerebrum) assuming $10^{-20} \mathrm{~J}$ per action potential, is within this range [10]. Considering there are in the order of $20 \cdot 10^{9}$ neurons in the cortices, this means that a fraction of a percent of the total neuronal capacity would match the energy associated with a coupling between earth oscillations and the cerebrum. The numbers of neurons are within the range of the critical mass that, depending upon the amount of recruitment of other neurons, could be below the threshold of "awareness" for the participant [11].

Annual oscillations in the amplitude of these slow waves have been reported around 230 $\mathrm{s}(4.3 \mathrm{mHz})$ and $270 \mathrm{~s}(3.7 \mathrm{mHz})$. Annual variations of background photon emissions of $\sim 10^{-}$ ${ }^{11} \mathrm{~W} \cdot \mathrm{m}^{-2}$ within the $3 \mathrm{mHz}$ range have also been measured [12]. The power associated with these energies at the fastest component of the 3 to $5.5 \mathrm{mHz}$ band $\left(5.5 \cdot 10^{-3} \mathrm{~s}^{-1}\right)$ would be $4.1 \cdot 10^{-12}$ and $10^{-14} \mathrm{~W}$ for the $70 \mathrm{~kg}$ body and the $1.5 \mathrm{~kg}$ cerebrum, respectively. Assuming 1 $\mathrm{m}^{2}$ cross-sectional area for the body and $10^{-2} \mathrm{~m}^{2}$ cross sectional area for the cerebrum, the flux densities would be $\sim 10^{-12} \mathrm{~W} \cdot \mathrm{m}^{-2}$.

Flux densities in the order of $10^{-11} \mathrm{~W} \cdot \mathrm{m}^{-2}$ are similar to photon densities that have been reliably measured from the right hemisphere of people sitting in complete darkness when they imagine light. The effect requires about 10 to $12 \mathrm{~s}$ to maximize and is reversible. The strength of the association between electroencephalographic power and the energy associated with photon emissions [13] from the brain is strong ( $\mathrm{rs} \sim 0.9$ ) and exhibits strong contributions from regions (the left prefrontal cortices). This functional association is consistent with the possibility that intentions or thoughts have clear physical bases with energies that are similar in magnitude to fundamental forces.

We reasoned that if gravitational and electromagnetic processes were coordinated within brain space these subtle changes could be coupled with proximal discrete physical chemical changes, specifically those involving electron tunneling across gaps that meet the criteria for the potential inclusion of Casimir forces. One device that utilizes this process is the Random Number Generator which has been employed for several decades to measure the effects of "intention" on random variation. The effects were so consistent over years, as shown by Jahn and Dunne [14], that they have been included as examples of classical subtle cerebral-environment effects within the Encyclopedia of Neuroscience [15].

If the operating mode is within the range of $\sim 10^{-20} \mathrm{~J}$, then any source of energy within this range, including electromagnetic energy $\left(\sim 10^{-19} \mathrm{~J}\right)$ either phase- or frequency-modulated within the dimensions of a plasma cell membrane $(10 \mathrm{~nm})$ would have the capacity to interact with these phenomena. Lunar distance ranges between $\sim 3.567 \cdot 10^{8} \mathrm{~m}$ (perigee) to $\sim 4.063 \cdot 10^{8}$ $\mathrm{m}$ (apogee). The gravitational effect from the moon $\left(7.33 \cdot 10^{24} \mathrm{~kg}\right.$ ) on a cell mass of $5.24 \cdot 10^{-13}$ $\mathrm{kg}$ would range from 15.52 to $20.14 \cdot 10^{-16} \mathrm{~N}$ and when applied across the width of a cell $\left(10^{-5}\right.$ $\mathrm{m})$ would range from 1.55 to $2.01 \cdot 10^{-20} \mathrm{~J}$. Considering the closer proximity of the latter to the energy associated with neuronal action potentials, $\sim 1.9 \cdot 10^{-20} \mathrm{~J}[2]$, we predicted that perigee would be particularly effective.

Here we present for the first time that the quantitative displacement from random of a dynamic process is strongly associated with the mass of the proximal person, strong modulations by changes in gravitational energies, and the capacity to modify this effect by applying experimentally generated weak magnetic fields over the temporal lobes. The most effective pattern which was designed to simulate long-term potentiation (LTP), the central process involved with consolidation of memory through the hippocampal formation, was within the 5 to $8 \mathrm{~Hz}$ range. These values are also within the range of the resonance frequency of the average cerebrum and the earth's circumference. The former is calculated by assuming 
a bulk velocity of about $4.5 \mathrm{~m} \cdot \mathrm{s}^{-1}$ [16] for the rostral-caudal waves generating the 20 to $25 \mathrm{~ms}$ intervals of consciousness and a circumference of about $60 \mathrm{~cm}$. The later is the traditional solution where the velocity of light is divided by $4 \cdot 10^{7} \mathrm{~m}$.

\section{METHODS}

\section{1. Subjects}

Participant age ranged from 22-52 years for $\mathrm{N}=15(\mathrm{~N}=7$ females, $\mathrm{N}=8$ males $)$. All were recruited from Laurentian University campus or the local community.

\section{2. Equipment}

Four solenoids (telephone pickup coils obtained from Radio Shack or The Source, Model: 44-533) were arranged over the approximate location of the temporal lobe of each hemisphere in a ' + ' pattern. Vertical solenoids were separated by $5 \mathrm{~cm}$, while the horizontal solenoids were separated by $8 \mathrm{~cm}$. The 4 solenoids were maintained in position on each side of a cap by Velcro. Shiva Technology software version $1 \mathrm{G}$ was designed by Professor Todd Murphy as part of the Shiva Neural Stimulation system (www.shaktitechnology.com/shiva/) was employed to generate the fields.

This technology employs signals based on patterns used in previous studies [17]. Patterned waveforms are converted into audio files, which were delivered from 4 USB soundcards to the solenoids attached to the cap. Each audio device sends its signal to two of the solenoids, splitting the left audio channel to one solenoid and the right channel to the other (e.g., audio device 1 controls solenoids 1 and 2, while device 2 controls solenoids 3 and 4). The resultant activation of the solenoid creates a weak-intensity magnetic field within the .04 to $.4 \mu \mathrm{T}$ range as verified by milligauss meters (AC Milligauss Meter Model UHS2, AlphaLab, Inc; www.trifield.com).

Random data was produced using a Psyleron REG-1 random event generator (www.psyleron.com). The device produced a random output which was generated by electron tunnelling effects within two field effect transistors. The varying voltage levels which result from this process were converted into digital data through a gated sampling procedure which allows for regularly spaced bit sequences.

The output of both transistors was internally compared through an XOR masking process (it uses an alternating 1/0 pattern to maintain complete randomness from chance expectation) in order to reduce any potential influence of physical artefacts or other external environmental variables.

The device itself was further protected from static electromagnetic factors by an aluminum outer shielding and a Permalloy mu-metal inner shield. Furthermore, the device was rigorously calibrated prior to shipment in order to ensure output conformed to statistical expectations. The random event generator (REG) was also tested in control experiments within our laboratory to confirm these expectations.

The resulting data stream was collected through USB-port using Psyleron FieldREG and Reflector software packages on a laptop computer. Data were produced at a rate of 1 event (200 0,1 bits/event) per second, with each event referring to the number of 1's out of 200 bits with binary probabilities, represented by a value of $0-200$.

The theoretical (chance) mean for each event is 100 with a standard deviation of $\sqrt{50}$. Statistical analyses were conducted using SPSS software v.17. 


\section{3. Procedure}

Prior to testing, participants viewed a short demonstration with the REG software in order to understand how they would be focusing their intention. No feedback was provided during testing. They were then seated in a comfortable chair in a dark acoustic chamber which was also a Faraday cage. During the experiment each subject wore the hat containing the 4 pairs of solenoids situated over the regions of the temporal lobes. The REG was placed approximately $1 \mathrm{~m}$ away at ground level on right side of the participant approximately $45^{\circ}$ from the plane of the forward line of sight.

Participants were first asked to intend for the REG data output to deviate either up or down for $\sim 5$ minutes. Following this, a $40 \mathrm{~Hz}$ 'chirp' signal was applied bilaterally through the Shiva software for 20 minutes as a 'primer' field. A physiologically-patterned 'target' field was then applied for 15 minutes before the participant was asked to again focus intention on the REG for 5 minutes while the field continued running. During this period $N=9$ participants received an EMF patterned after LTP (long-term potentiation) in the hippocampus over the right hemisphere.

The other $\mathrm{N}=6$ received an EMF patterned after burst-firing in the amygdala over the left hemisphere. Field assignment was randomized. REG data collection was hidden from the experimenter until testing had completed. The selection of these two patterns was based upon their efficacy revealed by previous research. In addition, there is evidence of differential responsivity of the two hemispheres. The right hemisphere appears to be particularly sensitive to small changes in global geomagnetic activity [18].

\section{4. Data Transformation}

Random Event Generator (REG) data was obtained for $\mathrm{N}=15$ participants during three conditions (pre-field, baseline, and field). For pre-field and field conditions (e.g., those involving conscious intention) all individual REG event scores were reversed where necessary in order to produce positive overall session deviations when output was shifted in the intended direction, and negative deviations for sessions which shifted in the direction opposite of intention.

This was accomplished by obtaining the deviation from the hypothetical mean (in bits) for each event score ( $x$-100) and multiplying by -1 , then adding 100 to the subsequent values, effectively reversing the overall trend to represent the direction of intention. All individual events were subsequently z-scored according to .5 chance expectations $([x-100] / \sqrt{50})$. Overall session $z$-scores were derived using Stouffer's method $\left(\sum z / \sqrt{n}\right)$, where $z=$ individual event $\mathrm{z}$-scores, and $\mathrm{n}=$ the number of events. Event sample sizes varied slightly between sessions, and therefore overall $z$-scores for each condition $\left(z_{c}\right)$ were obtained by first combining all individual event scores for all participants within a given condition (raw event values ranging from $0-200$ ).

The overall deviation from the natural mean (100) was computed $\left(\delta_{\mu}\right)$, along with the measurement uncertainty associated with the actual condition mean $\left(\sigma_{\mu}=\sigma / \sqrt{ } \mathrm{N}\right.$, where $\mathrm{N}=$ total number of events, and $\sigma=$ the natural standard deviation of $\sqrt{ } 50)$. These two values were then divided $\left(z_{c}=\delta_{\mu} / \sigma_{\mu}\right)$ in order to produce the z-scores and subsequent probabilities associated with each overall condition.

One-tailed probabilities are reported where z-scores are used for overall sessions or conditions involving participant intention (e.g., testing for intended direction). Comparisons between conditions were examined by obtaining the absolute difference between condition means (e.g., $\mu$ field $-\mu$ baseline), as well as the measurement uncertainty associated with this 
value given the specific event samples (e.g., $\sigma \cdot \sqrt{ }\left(\left[1 / \mathrm{N}_{\text {field }}\right]+\left[1 / \mathrm{N}_{\text {baseline }}\right]\right)$, where $\mathrm{N}=$ number of events, and $\sigma=\sqrt{ } 50)$. These values were then divided $\left(z_{c}=\delta_{\mu} / \sigma_{\mu}\right)$ in order to produce a $\mathrm{z}$-score used to determine the magnitude of the difference between conditions.

\section{RESULTS}

\section{1. Overall Condition Results}

During the baseline (no intention) condition the REG data conformed to statistical expectations $\left(z_{c}=-.6, p=.548\right)$. Results from the pre-field condition also did not significantly differ from chance $\left(z_{c}=1.406, p=.08\right)$.

However, the REG data from the field condition showed a significant deviation in the direction the participants had intended $\left(z_{c}=2.876, p=.002\right)$. The overall results for each condition are represented in Figure 1 as cumulating deviations. More detailed descriptive parameters for each condition are presented in Table 1 .

These values account for slight variations in event sample sizes between participants and subsequently for overall conditions. As shown in Table 2 the only significant overall difference between conditions revealed through this more precise process was between field and baseline conditions $\left(z_{c}=2.45, p=.007\right)$.

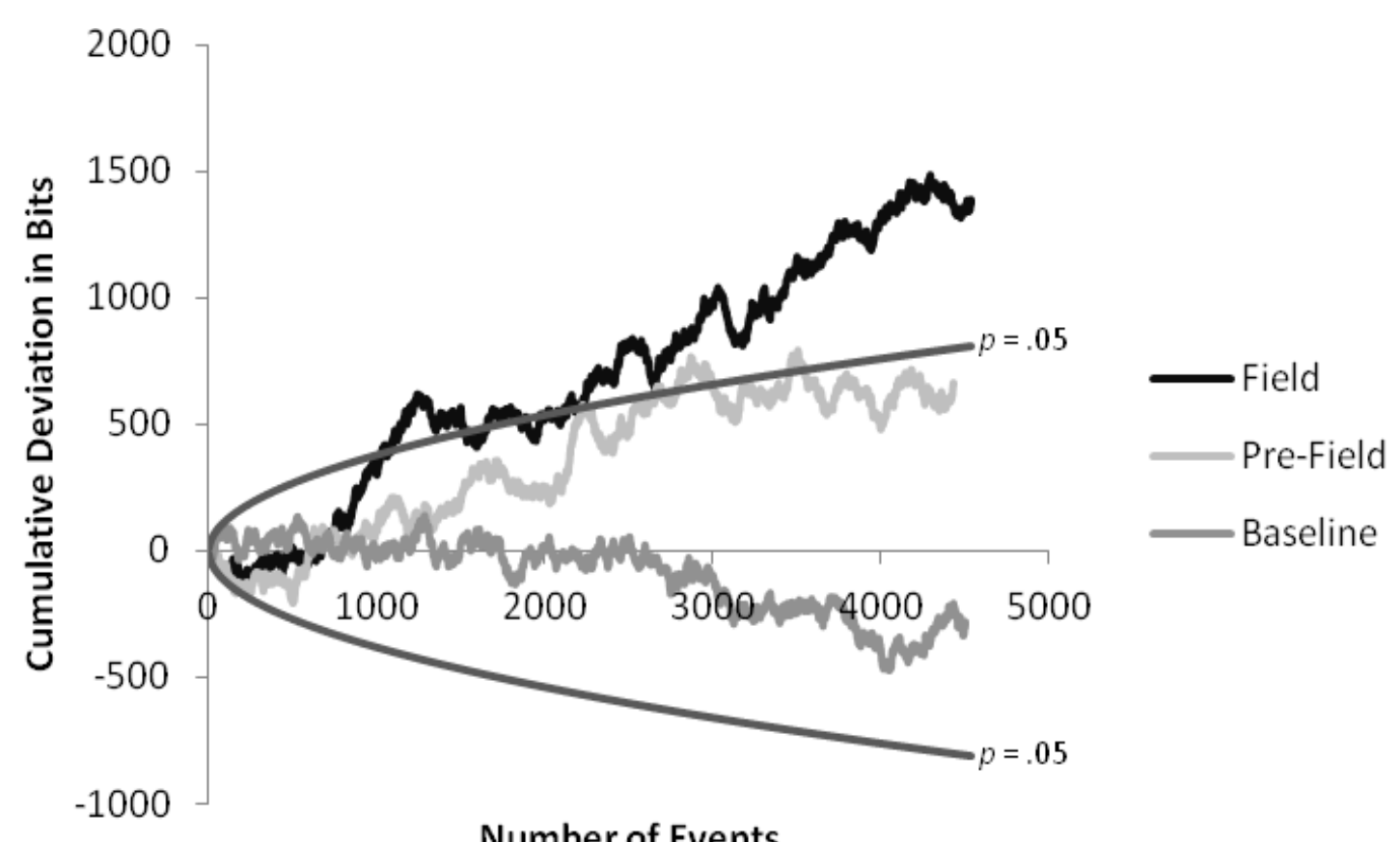

Figure 1. Combined cumulative deviations in REG output $1 \mathrm{~m}$ away for each condition; parabolas indicate threshold of statistical significance $(\mathrm{p}=.05$, one-tailed). The magnetic fields were applied over the temporal lobes of the participants. 
Table 1. Detailed REG results for overall conditions (Baseline, Pre-Field Intention, Intention with EMF application), data converted to directional measures (e.g., accounting for intended direction of deviations).

\begin{tabular}{cccc}
\hline Parameter & Baseline (BL) & Pre-Field & Field \\
\hline $\mathbf{N}$ & 4500 & 4433 & 4533 \\
$\boldsymbol{\mu}$ & 99.937 & 100.149 & 100.302 \\
$\boldsymbol{S} \boldsymbol{d}$ & 7.042 & 7.1 & 7.183 \\
$\boldsymbol{\sigma}_{\boldsymbol{s} \boldsymbol{d}}$ & .001 & .001 & .001 \\
$\boldsymbol{\delta}_{\boldsymbol{\mu}}$ & -.063 & .149 & .302 \\
$\boldsymbol{\sigma}_{\boldsymbol{\mu}}$ & .105 & .106 & .105 \\
$\boldsymbol{z}_{\boldsymbol{c}}$ & -.6 & 1.406 & $\mathbf{2 . 8 7 6}$ \\
$\boldsymbol{P}$ & $.548^{* *}$ & $.08 *$ & $\mathbf{. 0 0 2} *$ \\
$\boldsymbol{\%} \boldsymbol{I D}$ & $.467 \dagger$ & .667 & .733 \\
\hline
\end{tabular}

Parameter Key:

$\mathbf{N}$ : Number of events (200 bits/event)

$\boldsymbol{\mu}$ : Mean event score $(0-200)$

$\boldsymbol{s} \boldsymbol{d}$ : Standard deviation of REG event scores

$\sigma_{s d}:$ Measurement uncertainty in value of sd; $\sigma_{s d}=\sigma / \sqrt{2} \mathrm{~N}$, where $\sigma=\sqrt{50}$

$\boldsymbol{\delta}_{\mu}$ : Absolute deviation from theoretical chance expectations $(\mu-100)$

$\sigma_{\mu}$ : Measurement uncertainty in value of $\delta_{\mu} ; \sigma_{\mu}=\sigma / \sqrt{ } \mathrm{N}$, where $\sigma=\sqrt{50}$

$z_{c}$ : Overall condition z-score adjusted for measurement uncertainty; $z_{c}=\delta_{\mu} / \sigma_{\mu}$

$\boldsymbol{p}$ : Probability of $z_{c} * \& * *$

\%ID: Proportion of sessions with deviation in the intended direction; $\% I D=\mathrm{N} s$ with intention $/$

$\mathrm{N} s$, where $\mathrm{N} s=$ number of test sessions $\dagger$

$\dagger$ For directionality of baseline sessions, positive values are considered to be with intention.

*One-tailed probability (e.g., intention involved).

**Two-tailed probability (e.g., no intention).

Table 2. Detailed REG results comparing overall conditions, data converted to directional measures (e.g., accounting for intended direction of deviations).

\begin{tabular}{cccc}
\hline Parameter & PreField-BL & Field-BL & Field-PreField \\
\hline $\mathbf{N}$ & 8933 & 9033 & 8966 \\
$\boldsymbol{\delta}_{\boldsymbol{\mu}}$ & .212 & .365 & .153 \\
$\boldsymbol{\sigma}_{\boldsymbol{\mu}}$ & .15 & .149 & .149 \\
& & & \\
$\boldsymbol{z}_{\boldsymbol{c}}$ & 1.413 & $\mathbf{2 . 4 5}$ & 1.027 \\
$\boldsymbol{p}$ & $.079 *$ & $\mathbf{. 0 0 7} *$ & $.152 *$ \\
\hline
\end{tabular}

Parameter Key:

$\mathbf{N}$ : Combined number of events (200 bits/event)

$\boldsymbol{\delta}_{\mu}$ : Absolute deviation; e.g., $=\mu_{\text {field }}-\mu_{\text {baseline }}$

$\sigma_{\mu}$ : Measurement uncertainty in $\delta_{\mu}$; e.g., $=\sigma \cdot \sqrt{ }\left(\left[1 / \mathrm{N}_{\text {field }}\right]+\left[1 / \mathrm{N}_{\text {baseline }}\right]\right)$, where $\sigma=\sqrt{ } 50$

$z_{c}$ : z-score of overall difference adjusted for measurement uncertainty; $z_{c}=\delta_{\mu} / \sigma_{\mu}$

p: Probability of $z_{c}$ (one-tailed) 


\section{2. REG Differences between Field Treatments}

The overall deviation from chance for the nine subjects exposed to the hippocampal field condition was statistically significant (Fig. $2 ; z_{c}=3.067, p=.001$ ), with three independently significant sessions. All sessions in this condition deviated in the direction intended. However, results from the amygdaloid pattern condition were within chance expectations (Fig. $2 ; z_{c}=.79, p=.215$ ). Although there were two independently significant sessions within this condition, there were also two sessions which deviated in the direction opposite of intention.

Following further analyses (Table 3 ) it was discerned that the hippocampal field treatment significantly differed from the associated baseline results $\left(z_{c}=2.656, p=.004\right)$, while the amygdala treatment did not significantly differ from control measures $\left(z_{c}=.61, p=\right.$ $.271)$.

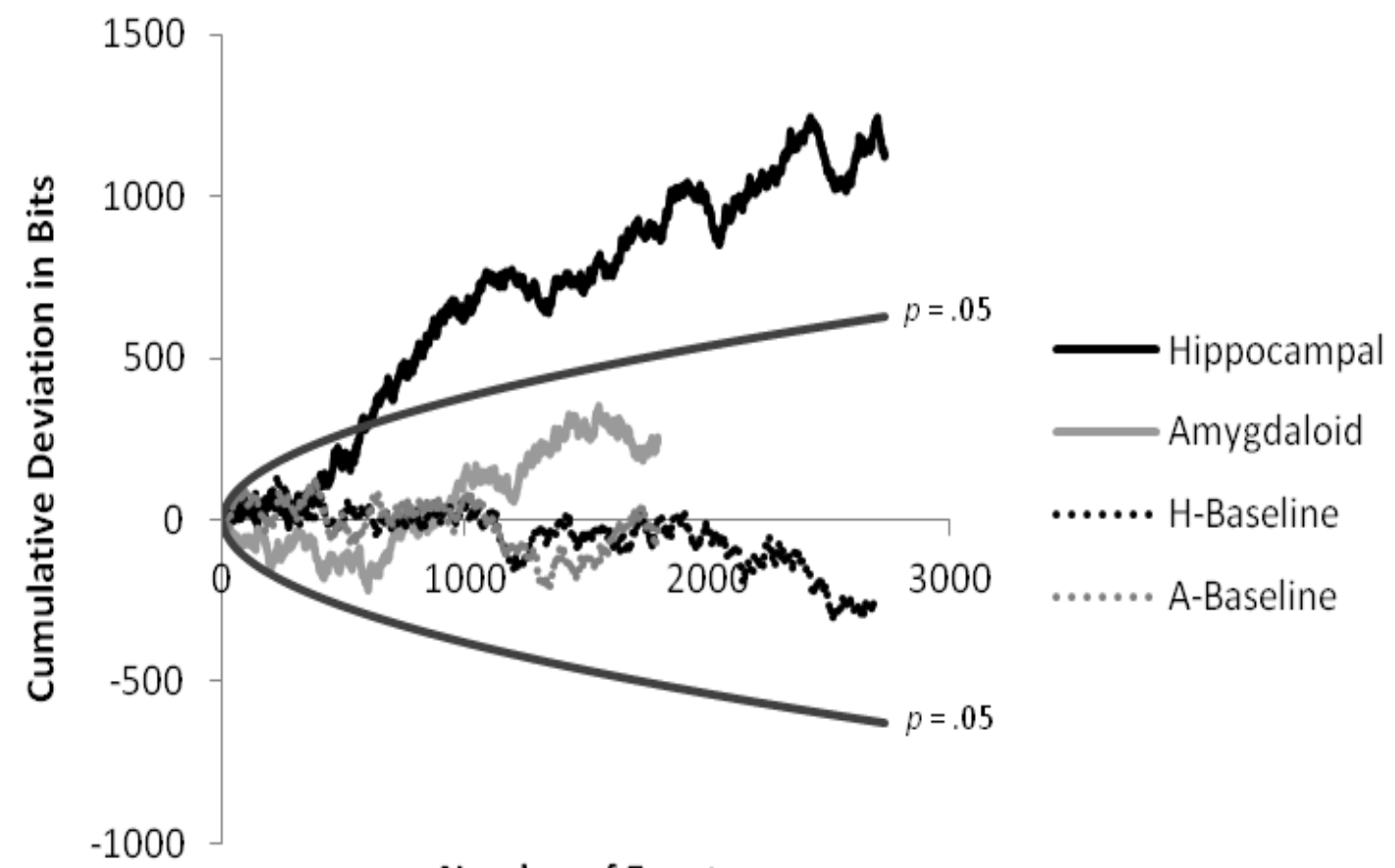

Number of Events

Figure 2. Combined cumulative deviations of REG output for each field condition and associated baseline measures; parabolas indicate threshold of statistical significance $(\mathrm{p}=.05$, one-tailed). The

"hippocampal" patterned magnetic field applied over the right temporal lobe during intention produced significant deviations.

Means, absolute means, and standard deviations were obtained for each minute (5) of REG output using data prior to directional transformation; sessions were not converted with respect to direction while z-scores were obtained from the raw data. An independent t-test revealed a statistically significant difference between field treatments (hippocampal and amygdaloid, respectively $\mu=.132, s d=.066$ and $\mu=.046, s d=.013$ ) for the absolute mean REG score during minute 3 . 
Table 3. Detailed REG results for each field condition (hippocampal and amygdaloid) with associated baseline measures; data converted to directional measures (e.g., accounting for intended direction of deviations).

\begin{tabular}{ccccc}
\hline Parameter & Hippocampus(Hi) & Amygdala(Am) & BL(Hi) & BL(Am) \\
\hline $\mathbf{N}$ & 2737 & 1796 & 2700 & 1800 \\
$\boldsymbol{\mu}$ & 100.414 & 100.132 & 99.904 & 99.988 \\
$\boldsymbol{S} \boldsymbol{d}$ & 7.253 & 7.075 & 7.031 & 7.06 \\
$\boldsymbol{\sigma}_{\boldsymbol{s} \boldsymbol{d}}$ & .096 & .118 & .096 & .118 \\
$\boldsymbol{\delta}_{\boldsymbol{\mu}}$ & .414 & .132 & -.096 & -.012 \\
$\boldsymbol{\sigma}_{\boldsymbol{\mu}}$ & .135 & .167 & .136 & .167 \\
$\boldsymbol{\sigma}_{\boldsymbol{c}}$ & $\mathbf{3 . 0 6 7}$ & .79 & -.706 & -.072 \\
$\boldsymbol{P}$ & $\mathbf{. 0 0 1} *$ & $.215^{*}$ & $.76 * *$ & $.529 * *$ \\
$\boldsymbol{\%} \boldsymbol{I D}$ & .889 & .5 & $.333 \dagger$ & $.667 \dagger$ \\
\hline
\end{tabular}

\section{Analysis of Potential Gravity Contributors}

To examine any potential influence of subtle changes in gravity on REG output during human intention paired with the additional energy from transcerebral EMF application, the weight of each participant $(\mathrm{kg})$ was obtained. The participant database was subsequently split by the year they were tested (2012 and 2013). Pearson and Spearman analyses indicated that participant weights in the test block from $2012(\mathrm{~N}=7)$ were significantly correlated with average REG scores during minute 1 of testing (Fig. 3; $r=-.805, p=.029 ; r=-.857, p=$ .014). Within the second block of testing $(\mathrm{N}=8)$, Spearman correlations revealed a significant relationship between weight and average REG output during minute 3 (Figure 4). Assuming the maximum value for a period, this would be equivalent to $16 \mathrm{mHz}$ to $5.5 \mathrm{mHz}(3$ min) which is well within the range predicted if there is a coupling between the earth's free oscillations, the person's mass, and the forces and energies available locally for the effect.

Although body weight of the participants was clearly associated with the deviations we pursued the hypothesis the interaction between the mass of the cell and the most obvious proximal changing source of small gravitational force, the moon, could contribute to the interaction between the cerebral activity associated with intention and a proximal (within $1 \mathrm{~m}$ ) random physical process. Subsequently, the numbers of days since the most recent lunar apogee and perigee were obtained for each session. Lunar data was derived from the Lunar Extremes database. Both parametric and non-parametric correlation analyses (Figure 5) revealed a significant relationship between perigee and the average REG score during minute $2(r=-.718, p=.003 ;$ rho $=-.774, p=.001)$.

In order to determine if this effect was further influenced by the mass, and therefore gravity, of the participant a partial correlation was completed between perigee and average REG score during minute 2 while controlling for participant weight. The significant correlation (Figure 6) was maintained and, furthermore, there was an increase in the strength of this relationship $(r=-.77, p=.001$; rho $=-.818, p<.001)$ when controlling for individual weight differences. Both perigee and average REG score were entered into separate linear regressions with weight to obtain the standardized residual values plotted in order to demonstrate the partial correlation. Subsequent correlation analyses revealed a significant relationship between the average REG score during minute 2 and the overall final session 
score $(r=.671, p=.006$; rho $=.70, p=.004)$. This would be consistent with the approach that the forces and energies associated with the second minute, the duration overlapping with the periodicity of earth oscillations, was central to affecting the overall outcome of the deviations from chance.

\section{Test Block 1 (2012)}

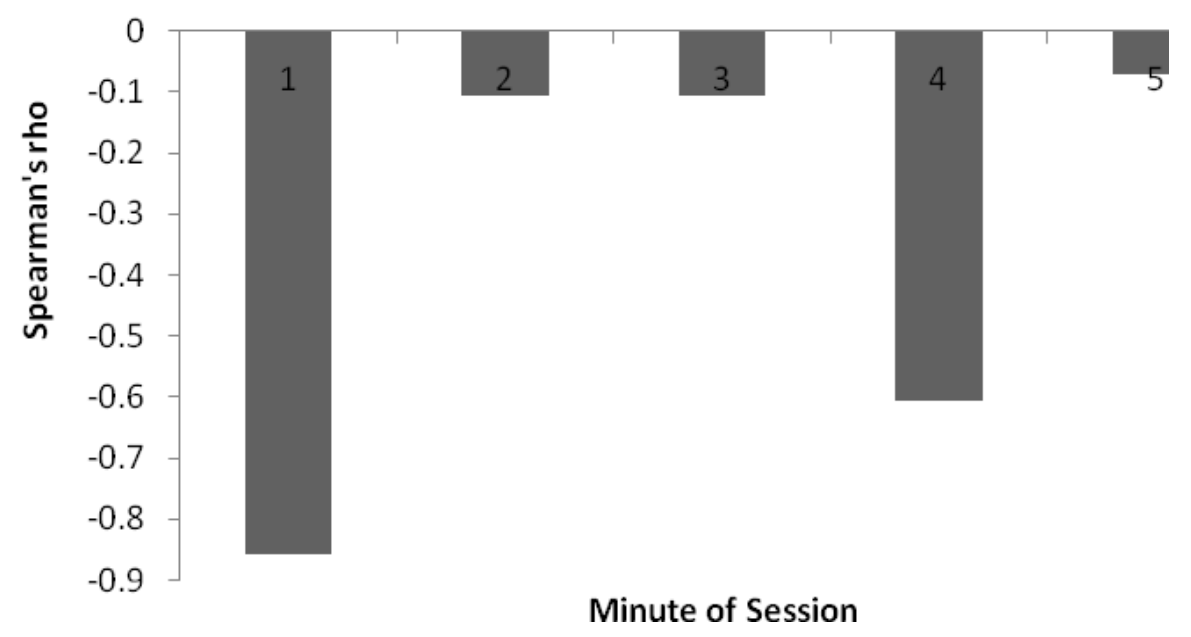

Figure 3. Spearman correlation values for participant weight and mean REG score in each minute of testing (2012).

\section{Test Block 2 (2013)}
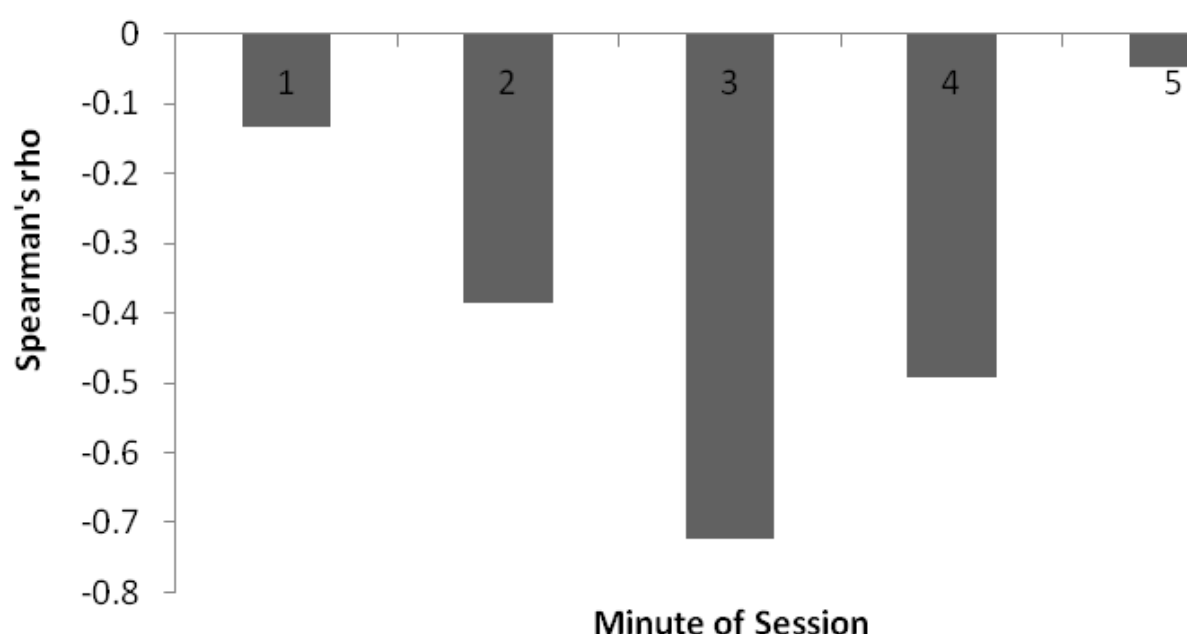

Minute of Session

Figure 4. Spearman correlation values for participant weight and mean REG score in each minute of testing (2013). 


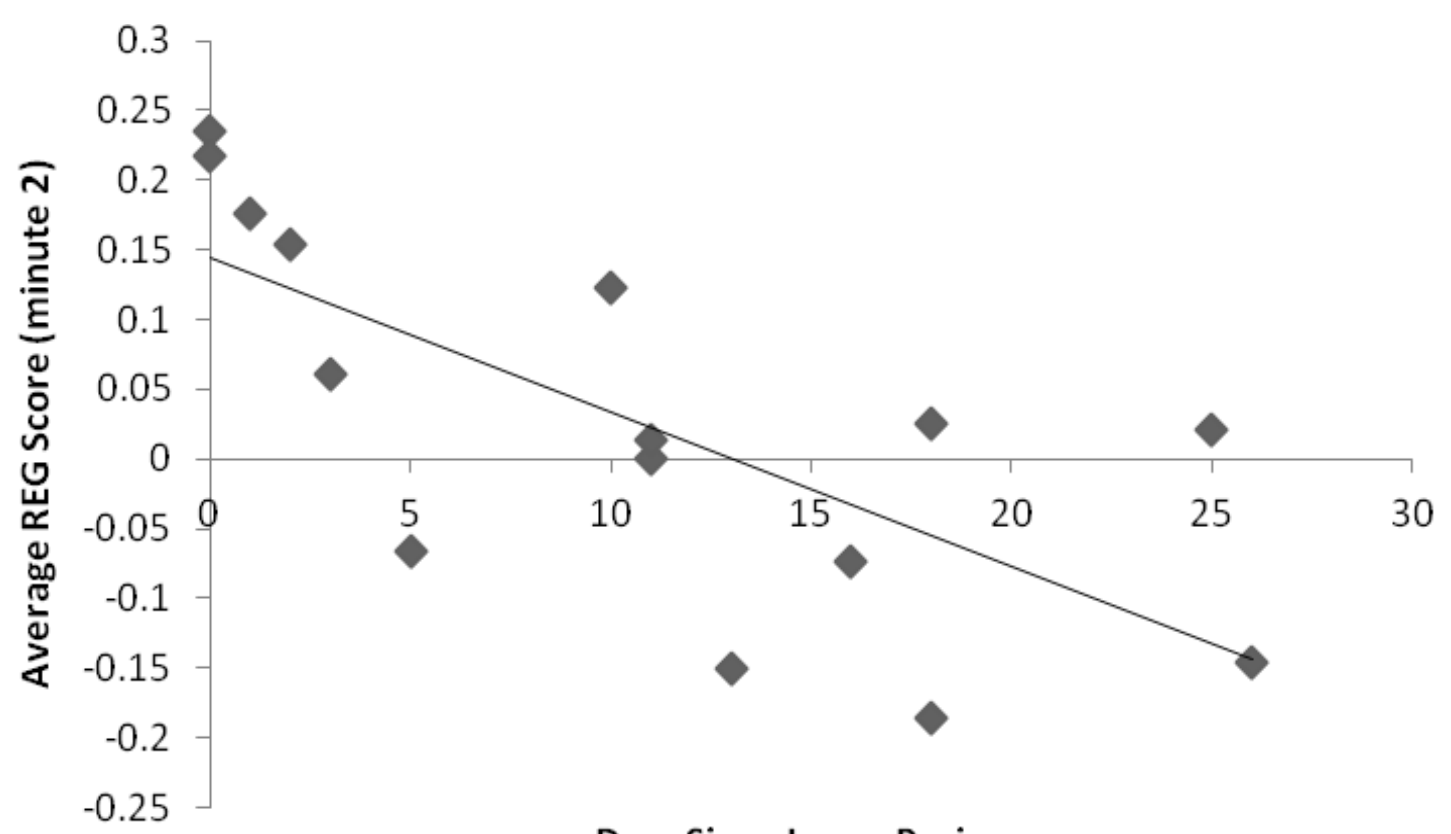

Days Since Lunar Perigee

Figure 5. Correlation between perigee values and average REG score during minute 2 .

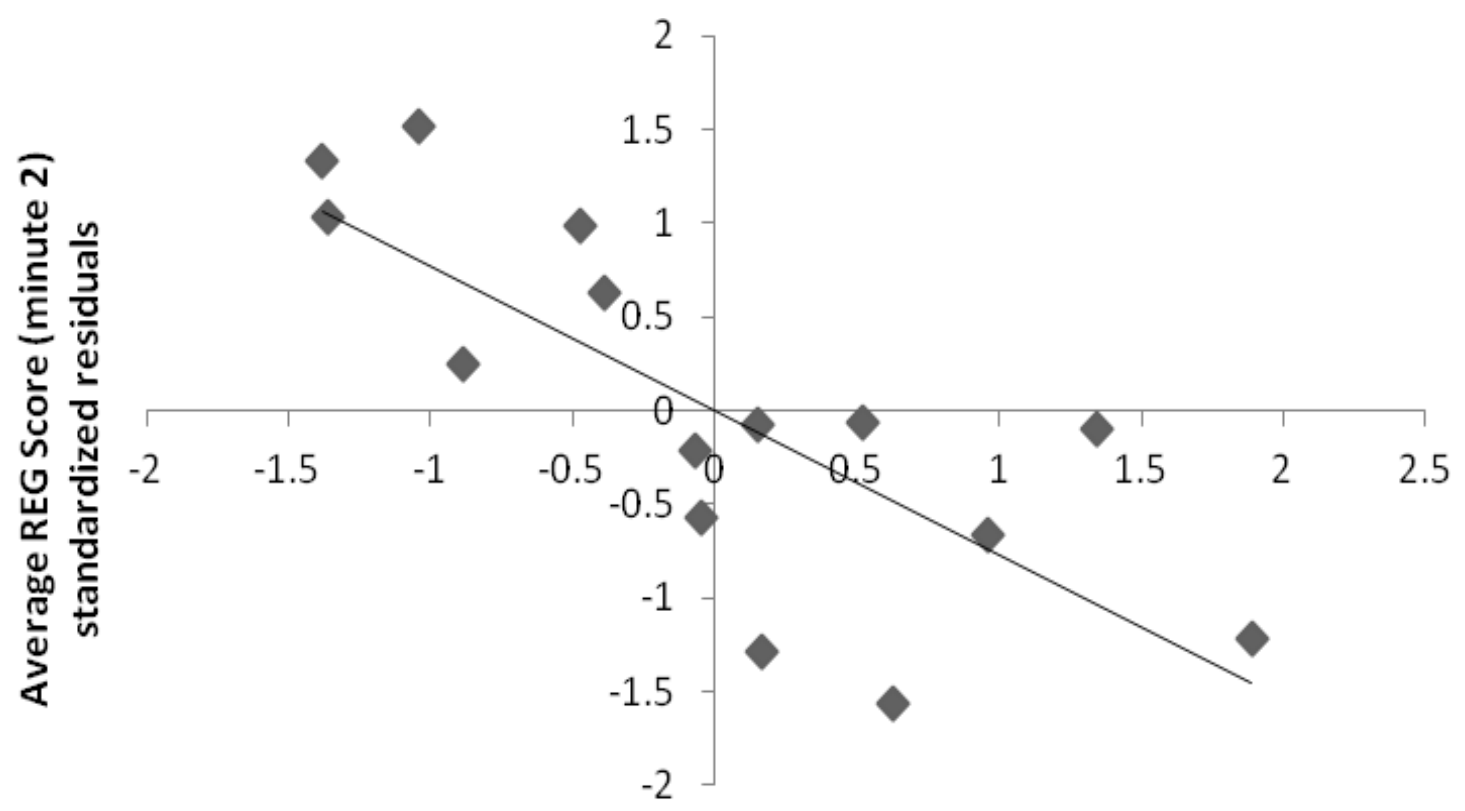

Time Since Lunar Perigee standardized residuals

Figure 6. Partial correlation between perigee values and average REG score during minute 2, controlling for participant weight; standardized residual values. 


\section{DISCUSSION AND CONCLUSIONS}

The results of this study may reflect one of the first interdisciplinary and integrative experiments that relate the quantitative values from theoretical relationships between the electromagnetic energies generated by cells and aggregates of cells with those associated with inferred or direct effects of gravity and gravitational-like forces. The consistency and convergence of the results emphasize the importance of multidisciplinary approaches to critical physical phenomena that include concepts and problems that relate astronomy, chemistry, and physics as well as their reflection within the biological sciences.

The concept of "random" is essential to all sciences and is the implicit assumption that drives the central limit theorem, that is, if an infinite number of means from random populations of numbers were plotted, they would display the normal distribution. The possibility that proximity to electronic devices that control electron tunneling in a "random" fashion can influence the outcome of the "random" occurrence of events as a function of the mass of the person suggests that local gravitational effects may be more relevant than previously assumed. The modulation of this effect by lunar distance, particularly when perigee was employed as the reference, supports this type of process.

The effect of an applied magnetic field upon the effectiveness by which the intent of the participant displaced the random variations from chance indicates that the phenomena are physically-based and can be easily affected by experimental methods. The magnetic field pattern [19] is known to affect the representation of experience (memory) within brain space which is expressed as specific changes in spine growth and dendritic patterns. In essence the 40 to $400 \mathrm{nT}$ intensity patterned magnetic fields that were generated primarily over the right temporal lobes of the participants markedly enhanced the effects of their intention upon the deviation from random variation of proximal $(1 \mathrm{~m})$ electron tunneling in a commercial device.

We suggest that the increment of energy or quantum that mediates this effect is $\sim 2 \cdot 10^{-20}$ J. This is the energy upon a unit charge $\left(1.6 \cdot 10^{-19} \mathrm{~A} \cdot \mathrm{s}\right)$ from a typical change in voltage during an action potential from a neuron. The unit of energy is also the implicit energy from the gravitational force from the earth upon a typical cell mass when applied across the plasma membrane and the functional energy from lunar gravitational effects around the time of perigee across the width of the typical neuronal soma. Such convergence allows the condition for the potential interaction, amplification, and dampening of these energies during dynamic processes.

The primary temporal component for the energy within the cerebral volume and the general body mass is likely to originate from the natural free oscillations from the earthatmospheric interface during seismic-free conditions [8]. The energy within the volume of the human cerebrum or average body mass would be in the order of $10^{-13} \mathrm{~J}$ to $10^{-11} \mathrm{~J}$, respectively. From this perspective the fact that our most effective changes from applied magnetic fields across the temporal lobes is usually between $40 \mathrm{nT}$ and $400 \mathrm{nT}$ is expected because comparable magnetic energies would be available within the cerebral volume from this range of intensities. In the present study this was sufficient, when the appropriate pattern was applied, to produce large deviations within the random number generators.

Why the presence of and intention by a human being should affect electron tunneling may be related to shared spatial features of the functional unit of the brain (the synapse) and the two surfaces across which electrons tunnel. The typical surface area of a synapse is in the order of 0.5 to $2 \mu \mathrm{m}^{2}$ and in the balance of probabilities this is the approximate width surface area from which the electrons tunnel within the RNG. The two "plates" of the synapse are 
separated by about $10 \mathrm{~nm}$ which is within an order of magnitude of the separation likely for the random number generators.

If this feature is relevant then a type of resonance could occur whereby energies are exchanged. The Casimir force within the brief period of neutrality for the geometry of a typical synapse is $\sim 0.52 \cdot 10^{-6} \mathrm{~N}$ and when applied over the distance of this separation $\left(10^{-8} \mathrm{~m}\right)$ is $\sim 0.52 \cdot 10^{-14} \mathrm{~J}$. When divided by Planck's constant to obtain the frequency the equivalent electromagnetic wavelength, assuming the velocity of light, is $38 \mathrm{pm}$. This is within measurement error of the radius of the hydrogen atom [20]. We think it may be relevant that a classic Cosic [21] velocity of an electron, $\sim 2 \cdot 10^{5} \mathrm{~m} \cdot \mathrm{s}^{-1}$ would be associated with mass-energy equivalence of $\sim 1.8 \cdot 10^{-20} \mathrm{~J}$. Hence the congruence between the energetic boundaries could allow for the conditions for non-locality. We [22] have shown previously that non-local effects, that is "entanglement" or "excess correlation" between two loci can occur for periods of about 8 min when they share similar shaped magnetic fields with specific rates of change in angular velocities.

\section{References}

[1] T. Borowski, International Letters of Chemistry, Physics and Astronomy 1 (2012) 1-5.

[2] M. A. Persinger, Current Medicinal Chemistry 17 (2010) 3094-3098.

[3] T. E. Decoursey, Physiological Reviews 83 (2003) 475-579.

[4] M. A. Persinger, S. A. Koren, G. F. Lafreniere, NeuroQuantology 6 (2008) 262-271.

[5] A. A. Minakov, A. P. Nikolaenko, L. M. Rabinovitch, Radiofizika 35 (1992) 915-923.

[6] B. M. Vladmirskii, Biophysics 40 (1995) 915-923.

[7] M. A. Persinger, The Open Astronomy Journal 5 (2012) 41-43.

[8] K. Nishida, N. Kobayashi, Y. Fukao, Science 287 (2000) 2244-2246.

[9] K. Saroka, M. A. Persinger, Epilepsy and Behavior 28 (2013) 395-407.

[10] M. A. Persinger, C. Lavallee, Journal of Consciousness Studies 19 (2012) 128-153.

[11] G. S. Berns, J. D. Cohen, M. A. Mintun, Science 276 (1997) 1272-1275.

[12] M. A. Persinger, International Journal of Geosciences 3 (2012) 192-194.

[13] B. T. Dotta, K. S. Saroka, M. A. Persinger, Neuroscience Letters 513 (2012) 151-154.

[14] R. G, Jahn and B. J. Dunne, "Margins of reality: the role of consciousness in the physical world”, Harcourt Brace Javanovich 1987.

[15] G. Adelman (ed), "Encyclopedia of neuroscience”, Birkhauser 1987.

[16] P. L. Nunez, "Neocortical dynamics and human EEG rhythms", Oxford Press 1995.

[17] M. A. Persinger, K. S. Saroka, S. A. Koren, L. S. St-Pierre, Journal of Consciousness Exploration and Research 1 (2010) 803-830.

[18] B. P. Mulligan, M. D. Hunter, M. A. Persinger, Advances in Space Research 45 (2010) 940-948.

[19] Q. H. Mach, M. A. Persinger, Brain Research 1261 (2009) 45-53. 
[20] M. A. Persinger, C F. Lavallee, Journal of Consciousness Exploration and Research 1 (2010) 785-807.

[21] I. Cosic, IEEE Transactions for Biomedical Engineering 41 (1994) 1101-1114.

[22] B. T. Dotta, M. A. Persinger, Journal of Biophysical Chemistry 3 (2012) 72-80. 